\title{
Screen Me, Smartphone! Using an AI-Screening Tool to Assist Underage Refugees in Recognizing Potential Traumatization
}

\author{
Lisa Mühl* \\ Rheinische Fachhochschule Köln \\ lisa.muehl@rfh-koeln.de
}

\author{
Aike C. Horstmann \\ Rheinische Fachhochschule Köln \\ aike.horstmann@web.de
}

\author{
André Wittenborn \\ Rheinische Fachhochschule Köln \\ andre.wittenborn@rfh-koeln.de
}

\author{
Dunja Storch \\ Rheinische Fachhochschule Köln \\ dunja.storch@rfh-koeln.de
}

\author{
Jarek Krajewski \\ Rheinische Fachhochschule Köln \\ jarek.krajewski@rfh-koeln.de
}

\begin{abstract}
Many refugees experience critical life events or traumatic injuries during their flights. Here, underaged, (un)accompanied refugees are a particularly vulnerable group. To date, there are insufficient support structures that recognize the specific demands and allow for careful and early identification of indicators of traumatization or behavioral problems. As an approach to counteract these deficits and support underage refugees, the TraM project investigates the potential of an AI-based screening tool providing indications of post-traumatic stress disorder via speech-emotion-recognition. A data collection for standardized learning data was conducted as a basis for the described screening module and the planned algorithms for automatic classification. We encountered several challenges such as insufficient data quality, uncertain classifications, and comorbidities such as depression as potentially confounding factors. Accordingly, emphasis lays on using the screening module for an initial examination of mental health and potential traumatization. This may encourage affected underage refugees to seek the help that is often highly needed.
\end{abstract}

\section{CCS CONCEPTS}

- Social and professional topics $\rightarrow$ User characteristics; Age; Adolescents; User characteristics; Race and ethnicity; • Computing methodologies $\rightarrow$ Artificial intelligence; Natural language processing; Speech recognition.

\section{KEYWORDS}

speech-emotion recognition, post-traumatic stress disorder, feature extraction, behavioral markers, refugee minors

\section{ACM Reference Format:}

Lisa Mühl ${ }^{*}$, Aike C. Horstmann, André Wittenborn, Dunja Storch, and Jarek Krajewski. 2021. Screen Me, Smartphone! Using an AI-Screening Tool to Assist Underage Refugees in Recognizing Potential Traumatization. In Mensch und Computer 2021 (MuC '21), September 05-08, 2021, Ingolstadt, Germany. ACM, New York, NY, USA, 5 pages. https://doi.org/10.1145/3473856.3474011

Permission to make digital or hard copies of all or part of this work for personal or classroom use is granted without fee provided that copies are not made or distributed for profit or commercial advantage and that copies bear this notice and the full citation on the first page. Copyrights for components of this work owned by others than ACM must be honored. Abstracting with credit is permitted. To copy otherwise, or republish, to post on servers or to redistribute to lists, requires prior specific permission and/or a fee. Request permissions from permissions@acm.org.

MuC '21, September 05-08, 2021, Ingolstadt, Germany

(C) 2021 Association for Computing Machinery.

ACM ISBN 978-1-4503-8645-6/21/09...\$15.00

https://doi.org/10.1145/3473856.3474011

\section{INTRODUCTION}

In recent years, many people from conflict-affected regions, particularly from Arabic-speaking countries, sought refuge in Germany. The necessity to leave their country of origin is frequently caused by traumatic injuries and critical life events [23]. Additionally, many report to have experienced traumatizing events during their flight [12] and thus are at a high risk of developing mental health disorders [19]. Some recent studies report robust epidemiological data and prevalence rates of mental health disorders of mainly adult refugees including post-traumatic stress disorder (PTSD) and depression [19]. For example, Tinghög et al. [22] reported prevalence rates of $29,9 \%$ for PTSD and 40,2 \% for depression in Syrian refugees who sought refuge in Sweden [19]. Another study by Cheung et al. [6] also examined Syrian refugees living in Sweden and Turkey and found a PTSD prevalence rate of $43 \%$ [19]. However, about half of the world's refugees are children and adolescents under the age of 18. The number of unaccompanied refugee minors in Germany has risen to over 60,000 in 2016 [25]. A review paper by Witt et al. [26] established a prevalence of mental health disorders in unaccompanied refugee minors ranging from 20 to $81.5 \%$ [25]. Therefore, accompanied and unaccompanied refugee minors represent a particularly vulnerable target group [18, 25], mainly because the strains and traumas often remain unnoticed [10, 13]. To date, there are not sufficient support structures that recognize the specific demands of refugee minors [9] and that allow for a careful and early identification of indicators for traumatization or behavioral problems. Further, there is a lack of qualified interventions and referral of the affected persons to appropriate contact points $[11,17,23]$.

Online-based initiatives such as information and support websites or apps are therefore a promising approach because they are low-threshold and do not require as many resources as face-to-face counseling services. However, these approaches still have their limitations in screening children and adolescents since it is difficult or impossible to specify their symptoms in a differentiated and precise manner with self-report-based scales [8]. The currently deficient screening methods could be improved by the integration of AIbased affective computing detection algorithms for mental health disorders [24]. Accordingly, rapid screening of early indicators of mental health disorders via primarily terminal-based speechemotion-recognition provides a crucial solution approach.

Since speech is an interesting marker for cost-effective, remotely, and non-invasive measurement of mental health disorders, there 
has been increasing interest in speech-based approaches [15]. Besides, another advantage of speech as behavioral marker is the possibility of generalization across languages due to comparable vocal anatomy [15]. In contrast to other psychiatric disorders such as depression, there is much less information about speech alterations in PTSD [16]. Nevertheless, some studies addressed the analysis of PTSD through speech-based markers in different contexts. Scherer et al. [21] found that those with PTSD respond to positive, negative, and neutral interview questions with more tense voice features and decreased vowel spaces [16,21]. Whereas Marmar et al. [16] concluded that features which are indicating more monotonous and slower speech, lower variability of tonality, and less activation in voice are associated with higher probabilities of PTSD. Broek et al. [5] reviewed two experimental designs regarding the optimal activation of PTSD-related voice parameters. As a result, a free narration of autobiographical, emotionally charged events was more effective than reading stress- or joy-provoking stories.

\subsection{Uniqueness of the Proposed Approach of the TraM Project}

Against this background, the research project TraM, funded by the German Federal Ministry for Education and Research, aims to develop a low-threshold, technology-based screening, support, and diagnostic concept for underage, (un)accompanied refugees in form of a smartphone application. Within the application, a screening tool is intended to provide early and falsifiable indications of post-traumatic stress disorder via an AI-based audio analysis. The analysis is carried out locally on the user device, e.g., a tablet or smartphone, in a playful interaction scenario suitable for the target group [14, 24]. The screening is an anonymous self-test in Arabic to examine whether there are signs that indicate traumatization. If signs of traumatization are detected, contact to help offers can be arranged directly if desired by the adolescent. Compared with previous self-report-based approaches, the added value lies in the low-threshold recording of initial indicators of mental irregularities. Thus, the goal here is not to develop a diagnostic tool but a screening tool that offers minor refugees a low-threshold approach to investigate their mental health. Furthermore, the application aims to connect underage refugees and trained peers with comparable migration histories and to provide culture-, language- and age-appropriate interaction materials. Primarily the application is being developed to be multilingual (German, English, and Arabic) to avoid potential language barriers.

Standardized learning data is needed as a basis for the described screening module and the planned algorithms for automatic classification. Since there is no adequate learning data of Arabic-speaking adolescence publicly available, the necessary data was collected within the project. Hence, the data collection was initially designed as a laboratory study. However, due to the COVID-19 pandemic, it needed to be conducted remotely.

The main issues addressed in this paper are the data collection, the evaluation of the descriptive data, the proposed explorative approach for the feature extraction as well as some preliminary results of the trained model. It concludes with some of the major challenges, lessons learned, and potential futures research directions associated with the suitability of speech-emotion-recognition to assist underage refugees in recognizing their potential traumatization.

\section{METHOD}

\subsection{Study Sample and Data Collection}

The study's target group included young people between the ages of 14 and 22 years who fled to Germany after 2014, speak Arabic as their native language, possess average German language skills, and have no diagnosed depression. The latter was set as an exclusion criterion for participation due to the high comorbidity between depression and PTSD to avoid confounds in the audio data. Furthermore, an adequate hearing and speaking ability was set as condition for participation. To ensure that the participants fulfilled all inclusion criteria, they were required to answer a screening questionnaire before head.

Participants were recruited primarily through associations, organizations, and shelters that work with (underage) refugees. In addition, participants were recruited via Facebook groups aimed at refugees. Potential participants were provided with information about the TraM project, the survey's purpose and procedure, and the data processing within the recruitment process. All information was available in German and Arabic. In addition, there was a separate information sheet in Arabic for the parents or legal guardians. Written informed consent was obtained from all study participants and respectively their parents or legal guardians. Within the project, a wide-ranging review of the ethical legal and social issues was conducted beforehand and guides the entire project plan.

The data collection comprised two parts. At first, participants were asked to fill out an online-based questionnaire in German via LimeSurvey. The questionnaire collected information about their demographic background such as age, gender, level of education, number of completed school years, and the length of their stay in Germany. In addition, participants were asked to provide some information about their language skills such as whether they grew up bilingual and speak standard Arabic or any Arabic dialect. Culturally sensitive self-assessment scales were used to measure the extent to which the participants showed signs of PTSD or depression (see chapter 2.2). The second part focused on the collection of the audio data and was also divided into two parts. First, the subjects were asked to describe twelve different emotion-provoking images in Arabic for about 90 seconds. Respectively three pictures are supposed to evoke an either happy, sad, anxious, or neutral emotional state. Each description was followed by a query about the participant's current emotional state on a ten-point Likert scale $(1=$ "not at all" to $10=$ "very much"). This was followed by free narrations of autobiographical, emotionally charged events, also in Arabic. Here, the participants were asked to describe situations in which they either felt happy, sad, or anxious. Each emotionprovoking narration was followed by a free narration of a neutral event and by an assessment of the participants' current emotional state. In total, six free speech recordings were collected.

Since the data collection had to be conducted remotely, examiners accompanied the entire data collection process via Vectera, a browser-based meeting platform. The speech data was recorded via the smartphones of the subjects. To achieve as standardized and low-noise recordings as possible, the participants received detailed 
instructions from the examiner. In addition, a test run took place before the actual data recording.

\subsection{Instruments}

2.2.1 Reaction of Adolescents to Traumatic Stress (RATS). PTSD was assessed with the Reaction of Adolescents to Traumatic Stress (RATS) questionnaire [2], a self-report instrument which is used to indicate whether the adolescents are at risk for developing PTSD $[2,3]$. The RATS was chosen because it is a reliable and valid screening instrument designed particularly for refugee adolescents [3] and thus an ideal fit for the present study sample. The questionnaire consists of 22 items rated on a 4-point Likert scale ranging from $1=$ "not too much" to 4 = "very much". The items are following the PTSD diagnostic criteria B, C, and D of the DSM-IV and are therefore divided into three clusters: intrusion (criteria B, items 1-6), numbing/avoidance (criteria C, items 7-15), and hyperarousal (criteria $\mathrm{D}$, items 16-22; [2,3]). To calculate a cut-off score for the training of the algorithm, the data must be transformed. In total, the data is divided into four scores. The sum scores can indicate severe (10-13), moderate (6-9), mild (3-6), and minimal (0-2) signs of traumatization [2]. The minimum score required to classify evidence of PTSD was set to 3 .

2.2.2 Beck Depression Inventory Fast Screen (BDI-FS). Depression was assessed with the Beck Depression Inventory Fast-Screen (BDIFS, [4]) which measures non-somatic symptoms of major depression based on the DSM-IV definition in adolescents and adults [20]. The BDI-FS consists of seven items related to the cognitive and affective symptoms sadness, loss of pleasure (anhedonia), suicide ideation, pessimism, past failure, self-dislike, and self-criticalness [20]. The sum scores can indicate serve (10-21), moderate (7-9), mild (4-6), and minimal (0-3) depression [20].

\section{PRELIMINARY DESCRIPTIVE RESULTS}

\subsection{Sociodemographic Characteristics and Language Skills}

During the survey, data of 50 participants were collected. Two datasets had to be excluded due to insufficient audio quality. Therefore, the final sample consists of 48 participants. The mean age of the participants in the present study was $19.0(S D=2.74)$. Most of the participants were male $(68.8 \%, \mathrm{n}=33)$ and had a Syrian citizenship $(87.5 \%, \mathrm{n}=30)$. Other countries of origin were Iraq $(2.1$ $\%)$, Lebanon (2.1\%), Kuwait (2.1\%), Palestine (2.1\%), and Egypt (2.1\%). The majority has been in Germany for 24 to 48 months $(70.8 \%, \mathrm{n}=48)$. More than half of the participants were students $(70.8 \%, \mathrm{n}=48)$ and more than a third have grown up bilingually or multilingually $(31.3 \%, \mathrm{n}=48)$. Arabic $(86.7 \%)$ was the most frequently learned first language, followed by English $(13.3 \%, \mathrm{n}=$ 33). Most of the participants spoke standard Arabic $(81.3 \%, \mathrm{n}=48)$ and Arabic dialects $(83.3 \%)$.

\subsection{Descriptive Values of Traumatization and Depression in the Sample}

The RATS and BDI-FS scores are highly correlated $(r=.508, p<$ .001). As shown in Table 1, most of the participants in the total sample are classified as score 1 , thus show no to minimal signs of
PTSD and are therefore below the cut-off score. One participant did not answer the self-report questionnaires.

Within the total sample, $51.1 \%$ are above the cut-off value of the BDI-FS and thus lie between the classification of mild to serve depression. Regarding gender distribution, there is only a slight difference. In comparison, more females than males are below the cut-off value and are thus classified as non-depressed as shown in Table 2.

\section{PRELIMINARY APPROACH OF ACOUSTIC FEATURE EXTRACTION AND CLASSIFICATION}

A total of 1180 audio (.wav) files of approximately 90-sec length each were collected. A complete data set of one participant consist of 18 files, twelve of which are image description files and six of which are free narrative files. A total of 36 complete data sets were collected and another 12 data sets were partially complete. The recordings were divided into two classes: no signs of traumatization (25 samples; 9 female, 16 male) and signs of traumatization (22 samples; 6 female, 16 male) with a threshold of 3, determined by the described cut-off score of the RATS. Due to data scarcity, it was only distinguished between the training and test set within a two-fold-cross-validation approach. As stated by Low et al. [15], a standardized feature extraction distributes several benefits since results of different studies can be compared with each other. Therefore, eGeMAPS is used, a set of 88 features which indicates voice changes during affective processes [15]. For the binary classification, a random forest classifier is applied.

\subsection{Preliminary Results of the Trained Model}

At this point, the evaluation and training of the model are still ongoing, so only preliminary results can be presented. The classification rates vary depending on which tasks are included in the training set. The highest accuracy of $73.2 \%$ (AUC $=0.682$ ) was achieved by including the descriptions of the emotion-provoking images happy, fearful, sad, and neutral. The lowest accuracy of $46.9 \%$ (AUC $=0.392$ ) was the result of including just the narration of an autobiographical sad event. In general, recordings from the picture descriptions tended to perform better than the free speech paradigm.

\section{DISCUSSION}

\subsection{Challenges of the Data Collection and Resultant Insights}

As mentioned earlier, there has been a deficiency in literature and research exploring the usage of speech-based emotion recognition in the context of PTSD [16]. As criticized by Marmar et al. [16], most published studies referring to this subject are based on small samples. Therefore, the challenge is to assess whether the proposed features related to PTSD are suitable for automatically predicting or diagnosing PTSD [16]. The present study also worked with a relatively small sample. However, it must be noted that this is a highly vulnerable sample that is also difficult to reach. In addition, the circumstances due to the current COVID-19 pandemic complicated the recruitment and data collection. Due to the remote 
Table 1: RATS cut-off score in the total sample and divided by gender

\begin{tabular}{|c|c|c|c|c|c|c|}
\hline & \multicolumn{2}{|c|}{ Total $^{\mathbf{a}}$} & \multicolumn{2}{|c|}{ Female $^{b}$} & \multicolumn{2}{|c|}{ Male ${ }^{c}$} \\
\hline & $\mathrm{n}$ & $\%$ & $\mathrm{n}$ & $\%$ & 1. $\mathrm{n}$ & $\%$ \\
\hline $0-2$ (minimal signs of traumatization) & 25 & 53.2 & 9 & 60.0 & 9 & 50.0 \\
\hline 3-5 (mild sings of traumatization) & 11 & 23.4 & 3 & 20.0 & 8 & 25.0 \\
\hline 6-9 (moderate sings of traumatization) & 6 & 10.64 & 3 & 20.0 & 2 & 6.3 \\
\hline 10-13 (serve signs of traumatization) & 5 & 12.77 & 0 & 0.0 & 6 & 18.8 \\
\hline
\end{tabular}

$\mathrm{n}^{\mathrm{a}}=47, \mathrm{n}^{\mathrm{b}}=15, \mathrm{n}^{\mathrm{c}}=32$

Table 2: BDI-FS total score in the total sample and divided by gender

\begin{tabular}{|c|c|c|c|c|c|c|}
\hline & \multicolumn{2}{|c|}{ Total $^{\mathrm{a}}$} & \multicolumn{2}{|c|}{ Female $^{b}$} & \multicolumn{2}{|c|}{ Male ${ }^{c}$} \\
\hline & $\mathrm{n}$ & $\%$ & $\mathrm{n}$ & $\%$ & $\mathrm{n}$ & $\%$ \\
\hline 0-3 (minimal depression) & 23 & 48.9 & 15 & 46.9 & 8 & 53.33 \\
\hline 4-6 (mild depression) & 10 & 21.3 & 8 & 25.0 & 2 & 13.33 \\
\hline 7-9 (moderate depression) & 10 & 21.3 & 6 & 18.8 & 4 & 26.67 \\
\hline 10-21 (serve depression) & 4 & 8.5 & 3 & 9.4 & 1 & 6.67 \\
\hline
\end{tabular}

$\mathrm{n}^{\mathrm{a}}=47, \mathrm{n}^{\mathrm{b}}=15, \mathrm{n}^{\mathrm{c}}=32$

setup and related factors such as background noises, different used recording devices, and an unstable internet connection, there could be significant fluctuations in the quality of the audio data. Nevertheless, the gathered data can be seen as ecologically valid since a subsequent screening tool would have to cope with noise and other environmental sounds as well. This will impact the further analysis of the collected data and the development of a robust classification model.

Since most subjects did not switch on their camera and had relatively poor German knowledge, communication between the examiner and the subjects was compromised. As a result, some participants had difficulties correctly carrying out the given tasks and needed several attempts. In addition, to check the audio quality, the examiner listened to the voice recordings while the participant filled out the self-assessment questionnaires. However, the possibilities to adjust the quality of the recordings were limited. Particularly background noises turned out to pose a frequent problem. Since many participants live with their families in a small space, the complete avoidance of background noises was hardly possible. Therefore, attention should also be paid to standardized recording devices provided to the participants, for example, by sending them appropriate devices in advance.

In general, one of the biggest challenges within the proposed approach is combining two relatively new research topics - AIbased recognition of PTSD and speech data in Arabic language. An issue that was not addressed enough in the present study was the challenge of the Arabic language and how cultural background influences emotional behavior and therefore vocalization [1]. The Arabic language differs from other languages such as German in various characteristics, particularly regarding the speech descriptors speed and energy. Thus, with regard to the wide variety of energy descriptions such as the fundamental pitch, the MFCCs,
RMS, and intensity, the Arabic language exhibits characteristic attributes which deviate from other languages [1]. As stated by Low et al. [15], an ideal model should be able to detect mental deviations or mental disorders even in new recording situations, regardless of age, gender, language, accent, or comorbidities present. Overall, a high degree of generalization should exist and be aimed for. It is important to note that while robustness can be considered the ultimate goal, in the context of the current study the characteristics of the Arabic language and its many dialects were significant obstacles. In future data collections focusing on the Arabic language, these factors should be considered. For example, larger samples could be collected, or the survey could focus on only one dialect such as Syriac Arabic. Nevertheless, the data corpus collected provides a first basis for an initial discussion on the use of speech data to identify PTSD in Arabic speaking refugee minors.

Moreover, the collected data shows a narrow range in terms of depression and trauma scores which was potentially insufficient for the development of a robust classification model. Within the sample, there are no clinically diagnosed persons. Also, as it was the case in other studies [16], the level of comorbidity with depression is high. Based on the self-assessment scales and the resulting scores, more participants can be categorized as depressed than traumatized. These results will make it difficult to determine whether the extracted features are actually indicative of PTSD. To avoid these potentially confounding effects, future studies should use a more extensive clinical sample in which people are examined who have been diagnosed with PTSD and the presence of depression has been ruled out. In order to develop truly robust classification algorithms, a non-clinical control sample should also be included in the experimental setup. 


\subsection{Conclusion}

Besides the frequently mentioned limitations regarding the use of speech-emotion-recognition in the context of PTSD, which is foremost the high comorbidity with depression [16], we faced several further challenges as described above. Accordingly, the approach of the TraM project deliberately focuses on the development of a screening rather than a diagnostic tool. Particularly within the vulnerable group of refugee minors, the focus is laid on a low-threshold access to support. In this context, the screening tool may be used for an initial examination of their mental health and any traumas they may have experienced in connection with their flight. Therefore, and under consideration of the challenges we faced, the emphasis has shifted to a screening tool to recognize deviations within the language which indicate mental health issues, independent of a clear distinction between depression and PTSD. This may prompt affected underage refugees to seek and get the help they need.

\section{ACKNOWLEDGMENTS}

The project "TraM - Understanding and Supporting Traumatized Minor Refugees: Development of A Low-threshold Screening and Support Portal as The Basis of a Comprehensive Psychosocial Diagnostic Model" is funded by the Federal Ministry of Education and Research (BMBF) and the funding program Forschung an Fachhochschulen (Duration: 01.09.2019-31.08.2022, Funding reference: 13FH005SB7).

\section{REFERENCES}

[1] Ajith Abraham, Ed. 2015. Proceedings of the 2015 15th International Conference on Intelligent Systems Design and Applications (ISDA). Marrakesh, Morocco, December 14-16, 2015. IEEE, Piscataway, NJ

[2] Tammy Bean, Ilse Derluyn, Elisabeth Eurelings-Bontekoe, Eric Broekaert, and Philip Spinhoven. 2006. Validation of the multiple language versions of the Reactions of Adolescents to Traumatic Stress questionnaire. Journal of traumatic stress 19, 2, 241-255. DOI: https://doi.org/10.1002/jts.20093.

[3] Tammy Bean, Elisabeth Eurelings-Bontekoe, Ilse Derluyn, and Philip Spinhoven 2004. Recation of Adolescents to Traumatic Stress questionnaire (RATS). User's Manual (2004). Retrieved from http://www.centrum45.nl/research/amaenggz/ ukamtool.php.

[4] Aaron T. Beck, Robert A. Steer, and Greogry K. Brown. 2013. Beck DepressionsInventar - FS. Deutsche Bearbeitung von Sören Kliem \& Elmar Brähler. Manual (1. Auflage). Persons Assessment, Frankfurt am Main.

[5] Egon L. Broek, Frans Sluis, and Ton Dijkstra. 2011. Telling the Story and Re-Living the Past: How Speech Analysis Can Reveal Emotions in Post-traumatic Stress Disorder (PTSD) Patients. In Sensing Emotions, Joyce Westerink, Martijn Krans and Martin Ouwerkerk, Eds. Philips Research Book Series. Springer Netherlands, Dordrecht, 153-180. DOI: https://doi.org/10.1007/978-90-481-3258-4_10.

[6] Man Cheung Chung, Nowf AlQarni, Mariam AlMazrouei, Shamsa Al Muhairi, Mudar Shakra, Britt Mitchell, Sara Al Mazrouei, and Shurooq Al Hashimi. 2018 The impact of trauma exposure characteristics on post-traumatic stress disorder and psychiatric co-morbidity among Syrian refugees. Psychiatry research 259 , 310-315. DOI: https://doi.org/10.1016/j.psychres.2017.10.035.

[7] Alexandra C. Constantinescu, Maria Wolters, Adam Moore, and Sarah E. MacPherson. 2017. A cluster-based approach to selecting representative stimuli from the International Affective Picture System (IAPS) database. Behavior research methods 49, 3, 896-912. DOI: https://doi.org/10.3758/s13428-016-0750-0.

[8] Jessica Deighton, Tim Croudace, Peter Fonagy, Jeb Brown, Praveetha Patalay, and Miranda Wolpert. 2014. Measuring mental health and wellbeing outcomes for children and adolescents to inform practice and policy: a review of child self-report measures. Child and adolescent psychiatry and mental health 8,14 .
DOI: https://doi.org/10.1186/1753-2000-8-14

[9] Deutscher Caritasverband e. V. 2016. Fluchtpunkte intern. Deutscher Caritasverband, Abteilung Soziales und Gesundheit, Referat Migration und Integration, Freiburg.

[10] Silke B. Gahleitner. 2012. Übergänge gestalten, Lebenskrisen begleiten. Klinische Sozialarbeit. Beiträge zur psychosozialen Praxis und Forschung, Bd. 4. Psychiatrie Verlag, Bonn

[11] Stephan Hiller, Michael Macsenaere, and Thomas Köck. 2018. Evaluation zu Unbegleiteten Minderjährigen Flüchtlingen. Lambertus Verlag.

[12] Matthew Hodes, Daljit Jagdev, Navin Chandra, and Anna Cunniff. 2008. Risk and resilience for psychological distress amongst unaccompanied asylum seeking adolescents. Journal of child psychology and psychiatry, and allied disciplines 49 , 7, 723-732. DOI: https://doi.org/10.1111/j.1469-7610.2008.01912.x.

[13] Heiner Keupp. 1997. Ermutigung zum aufrechten Gang. Forum für Verhaltenstherapie und psychosoziale Praxis, Bd. 35. Deutsche Gesellschaft für Verhaltenstherapie, Tübingen.

[14] Jarek Krajewski, Sebastian Schnieder, David Sommer, Anton Batliner, and Björn Schuller. 2012. Applying multiple classifiers and non-linear dynamics features for detecting sleepiness from speech. Neurocomputing 84, 65-75. DOI: https: //doi.org/10.1016/j.neucom.2011.12.021.

[15] Daniel M. Low, Kate H. Bentley, and Satrajit S. Ghosh. 2020. Automated assessment of psychiatric disorders using speech: A systematic review. Laryngoscope investigative otolaryngology 5, 1, 96-116. DOI: https://doi.org/10.1002/lio2.354.

[16] Charles R. Marmar, Adam D. Brown, Meng Qian, Eugene Laska, Carole Siegel, Meng Li, Duna Abu-Amara, Andreas Tsiartas, Colleen Richey, Jennifer Smith, Bruce Knoth, and Dimitra Vergyri. 2019. Speech-based markers for posttraumatic stress disorder in US veterans. Depression and anxiety 36, 7, 607-616. DOI: https://doi.org/10.1002/da.22890.

[17] Eva Möhler, Michael Simons, Michael Kölch, Beate Herpertz-Dahlmann, Michael Schulte-Markwort, and Jörg M. Fegert. 2015. Diagnosen und Behandlung (unbegleiteter) minderjähriger Flüchtlinge. Zeitschrift fur Kinder- und Jugendpsychiatrie und Psychotherapie 43, 6, 381-383. DOI: https://doi.org/10.1024/14224917/a000380.

[18] Birgit Möhrle, Claudia Dölitzsch, Jörg M. Fegert, and Ferdinand Keller. 2016. Verhaltensauffälligkeiten und Lebensqualität bei männlichen unbegleiteten minderjährigen Flüchtlingen in Jugendhilfe einrichtungen in Deutschland. Kindheit und Entwicklung 25, 4, 204-215. DOI: https://doi.org/10.1026/0942-5403/a000206.

[19] Y. Nesterko, D. Jäckle, M. Friedrich, L. Holzapfel, and H. Glaesmer. 2019. Prevalence of post-traumatic stress disorder, depression and somatisation in recently arrived refugees in Germany: an epidemiological study. Epidemiology and psychiatric sciences 29, e40. DOI: https://doi.org/10.1017/S2045796019000325.

[20] Helen Poole, Ros Bramwell, and Peter Murphy. 2009. The utility of the Beck Depression Inventory Fast Screen (BDI-FS) in a pain clinic population. European journal of pain (London, England) 13, 8, 865-869. DOI: https://doi.org/10.1016/j. ejpain.2008.09.017.

[21] Stefan Scherer, Gale M. Lucas, Jonathan Gracht, Albert S. Rizzo, and LouisPhilippe Morency. 2016. Self-Reported Symptoms of Depression and PTSD Are Associated with Reduced Vowel Space in Screening Interviews. IEEE Trans. Affective Comput. 7, 1, 59-73. DOI: https://doi.org/10.1109/TAFFC.2015.2440264.

[22] Petter Tinghög, Andreas Malm, Charlotta Arwidson, Erika Sigvardsdotter, Andreas Lundin, and Fredrik Saboonchi. 2017. Prevalence of mental ill health, traumas and postmigration stress among refugees from Syria resettled in Sweden after 2011: a population-based survey. BMJ open 7, 12, e018899. DOI: https://doi.org/10.1136/bmjopen-2017-018899.

[23] UNHCR - United Nations High Commissioner for Refugees. 2017. Global Trends: Forced Displacement 2016 (2017). Retrieved from http://www.unhcr.org/dach/wpcontent/uploads/sites/27/2017/06/GlobalTrends2016.pdf.

[24] Michel Valstar, Björn Schuller, Jarek Krajewski, Roddy Cowie, and Maja Pantic, Eds. 2014. Proceedings of the 4th International Workshop on Audio/Visual Emotion Challenge - AVEC '14. ACM Press, New York, New York, USA. DOI: https://doi.org/10.1145/2661806.

[25] Marco Walg, Ewgeni Fink, Mark Großmeier, Miguel Temprano, and Gerhard Hapfelmeier. 2017. Häufigkeit psychischer Störungen bei unbegleiteten minderjährigen Flüchtlingen in Deutschland. Zeitschrift fur Kinder- und Jugendpsychiatrie und Psychotherapie 45, 1, 58-68. DOI: https://doi.org/10.1024/14224917/a000459.

[26] Andreas Witt, Miriam Rassenhofer, Jörg M. Fegert, and Paul L. Plener. 2015. Hilfebedarf und Hilfsangebote in der Versorgung von unbegleiteten minderjährigen Flüchtlingen. Kindheit und Entwicklung 24, 4, 209-224. DOI: https: //doi.org/10.1026/0942-5403/a000177. 